\title{
The effect of short-term intensive insulin therapy in newly-diagnosed Type-2 diabetic patients
}

\author{
Cengiz Karacaer ${ }^{1}$, Taner Demirci², Hasret Cengiz ${ }^{3}$, \\ Ceyhun Varim ${ }^{4}$, Ali Tamer
}

\begin{abstract}
Objectives: We aimed to determine the effect of short-term intensive insulin therapy (SIIT) on long-term glycemic control in newly-diagnosed Type-2 diabetes mellitus (nT2DM) patients.

Methods: In this retrospectively study conducted at Sakarya University Medical Faculty Training and Research Hospital Outpatient Clinic between 2016 and 2019, 65 nT2DM patients were enrolled soon after their SIIT was initiated and were followed for at least a year. Intensive insulin treatment was discontinued after three or 12 months in a total of 65 (23-73-year-old) patients who had been newly diagnosed with T2DM. Intensive insulin therapy was discontinued when glycemic control and the target Glycated Hemoglobin (HbA1c) level had been attained, after which oral anti-diabetic drug (OAD), long-term insulin, and diet therapies were pursued.

Results: There was a significant decrease in mean HbA1c from $11.25 \pm 1.96 \%$ to $6.67 \pm 1.07 \%$. Fasting plasma glucose (FPG) was found to be an independent predictor of whether intensive insulin therapy could be discontinued after three months in a model that included FPG, $\mathrm{HbA1C}$, and body mass index measured at baseline. Patients with FPG $>13.8 \mathrm{mmol} / \mathrm{L}$ were 7.6 times more likely to require intensive insulin therapy beyond three months. There were significant decreases in HbA1c and low-density lipoprotein-cholesterol concentration, but no change in C-peptide between baseline and 3 months of therapy.

Conclusion: These results demonstrate that in nT2DM patients, intensive insulin therapy could be effective on long-term glycemic control and high FPG prior to three months of SIIT may predict poor long-term glycemic control.
\end{abstract}

KEYWORDS: Intensive insulin, Diabetes mellitus, Glycemic control, Beta-cell recovery, HbA1c.

doi: https://doi.org/10.12669/pjms.37.7.4013

How to cite this:

Karacaer C, Demirci T, Cengiz H, Varim C, Tamer A. The effect of short-term intensive insulin therapy in newly-diagnosed Type-2 diabetic patients. Pak J Med Sci. 2021;37(7):1972-1978. doi: https://doi.org/10.12669/pjms.37.7.4013

This is an Open Access article distributed under the terms of the Creative Commons Attribution License (http://creativecommons.org/licenses/by/3.0), which permits unrestricted use, distribution, and reproduction in any medium, provided the original work is properly cited.

\section{INTRODUCTION}

Type-2 diabetes mellitus (T2DM) is caused by an insulin deficiency that develops following chronic progressive beta-cell dysfunction. To prevent the deleterious effects of glucotoxicity and lipotoxicity

Correspondence:

Cengiz Karacaer, MD.

Adnan Menderes Caddesi,

Saglik Sokak, No: 195-54000,

Adapazari/Sakarya, Turkey.

E-mail: karacaerc@yahoo.com

* Received for Publication:

* Revision Received:

* Revision Accepted:
December 14, 2020

June 2, 2021

June 12, 2021 on pancreatic beta cells, it is necessary to act to preserve the endogenous functionality of the pancreas as early as possible. ${ }^{1-3}$

An increase in circulating C-peptide concentration during the early stage of insulin therapy is the most important indicator of improvement in the endogenous beta-cell reserve. ${ }^{3}$ However, there have been few studies in which control was shown to be achieved using shortterm intensive insulin therapy (SIIT) from as early as the first three months following diagnosis. ${ }^{4-6}$

A systematic review and meta-analysis recently demonstrated that 2-8 weeks of early, intensive insulin therapy can induce glycemic control, and after its discontinuation, patients can subsequently 
maintain glycemic control without anti-diabetic medication for up to 1-2 years. ${ }^{7}$

Reliable determinants of long-term glycemic control have yet to be determined. Therefore, we aimed to investigate the effects of SIIT on longterm glycemic control in newly diagnosed T2DM patients in this retrospective study.

\section{METHODS}

The total number of patients followed up in the polyclinic was 4800 , the number of patients with nT2DM was 122, and the number enrolled in the study was 65 . In a study involving 65 patients with diet, exercise, OAD, and various insulin treatment modalities, patients treated with short-term intensive insulin therapy (SIIT) were included in the study. Patients diagnosed with T2DM according to the World Health Organization diagnostic criteria (1999) were selected for the study. Patients who applied to Sakarya University Medical Faculty Training and Research Hospital Outpatient Clinic between 2016 and 2019 and who had not received any diabetes treatment before were selected from the file records.

Inclusion criteria:

1. 23-73 years old

2. Non-pregnant Type-2 diabetes mellitus

3. Have not had any previous diabetes treatment

4. Glycated Hemoglobin $(\mathrm{HgbA1c})>8$

5. Patients who were diagnosed with T2DM in the Diabetes Outpatient Clinic and came to the controls at three and 12 months

\section{Exclusion criteria:}

1. Previous diabetes therapy,

2. Chronic renal or hepatic failure

3. Current malignancy

4. Chronic infection

5. Acute or severe chronic diabetic complications or other severe comorbidity

6. Pregnancy

7. Type-1 diabetes mellitus.

The study protocol was approved by the Ethics Committee of Sakarya University Medical Faculty and was conducted in accordance with the principles of the Declaration of Helsinki (71522473/050.01.04/166).

In this retrospective study patient data were collected from Diabetes Outpatient Clinic records. Demographic data, family history, chronic diseases, and medications were recorded from patient files retrospectively. Routine biochemical measurements made at baseline, including serum lipids and $\mathrm{HbA1c}$, were recorded. Basic anthropometric measurements made using the Tanita TBF 300 device were recorded from the files. Laboratory tests previously performed by the patients in the Central Clinical Laboratory of Sakarya University Medical Faculty, Training and Research Hospital diabetes outpatient's clinic were recorded from their files. In our diabetes outpatient clinic, all patients are given training programs on self-management of diabetes, including sports, lifestyle changes and food intake guidance.

Intensive insulin therapy: Patients considered to be undergoing SIIT to reach their glycemic target (FPG 4.4-6.0 mmol/L, 2-hour post-prandial plasma glucose [PPG]: 4.4-7.8 mmol/L) were included in the study. Insulin Lispro (Humalog Kwikpen, Eli Lilly and Company, USA) or Insulin Aspart (Novorapid Flexpen, NovoNordisk, Bagsværd, Denmark) were the fast-acting analog insulins used. Insulin Glargine (Lantus Solostar, Sanofi Aventis, France) and Insulin Detemir (Levemir Flexpen Novo Nordisk, Bagsvaerd, Denmark) were the basal long-acting insulins used. Patients who administered a total daily insulin dose of $0.4-0.5$ $\mathrm{IU} / \mathrm{kg}, 50 \%$ of which was long-acting basal insulin and $50 \%$ fast-acting analog insulin were included in the study. The participants were taught to inject their short-acting insulin before each meal and their intermediate-acting insulin at bedtime. The type of insulin used was not included in the analysis.

Blood sampling and measurement: In order to determine the ideal insulin dose required, a total of six blood glucose measurements were taken in the first few days, three before and three after meals ( 2 hours after the meal) in the basal bolus regimen of capillary glucose concentration. FPG, PPG, and $\mathrm{HbA1c}$ were measured monthly for three months after the cessation of SIIT. FPG 4.4-7.2 mmol and PPG 10 or $<10 \mathrm{mmol} / 1$ were the targets for glycemic control, which are consistent with the American Diabetes Association (ADA) 2019 guidelines. All laboratory tests were performed in the Central Clinical Laboratory of Sakarya University Medical Faculty, Training and Research Hospital.

Statistical Analysis: IBM SPSS Statistics version 23.0 (Armonk, NY, USA) was used for statistical analyses. Power analysis was applied. The Kolmogorov-Smirnov test was used to determine whether data distributions were normal. Nonnormally distributed continuous data are presented using medians and interquartile ranges [IQRs]. Qualitative data are expressed as frequencies and percentages. The Friedman test was used to compare biochemical data among the three time 
points, and the Wilcoxon test was used for binary comparisons. The relationships between continuous variables were evaluated using multiple linear regression and Spearman correlation. Relationships between categorical variables were evaluated using multiple logistic regression (LR). Receiver operating characteristic (ROC) curve analysis was performed to determine the optimal cut-off for FPG. $P<0.05$, was regarded as representing statistical significance.

\section{RESULTS}

Sixty-five patients (41 men and 24 women), were recruited. Their mean age was $48.03 \pm 10.78$ (range $23-73$ years). The mean FPG was $15.3 \pm 6.1 \mathrm{mmol} / \mathrm{L}$ mean $\mathrm{HbA} 1 \mathrm{c} 11.3 \pm 1.96 \%$, mean BMI $30.4 \pm 5.8$ $\mathrm{kg} / \mathrm{m}^{2}$, respectively. All other laboratory and anthropometric data are presented in Tables-I \& II.

Basically, the fasting plasma glucose (FPG) was 15.3 [10.8 - 18.8] and PPG was 19.7 [14.8 - 23.3]. Treatment with SIIT significantly reduced FPG and PPG at $3^{\text {rd }}$ and $12^{\text {th }}$ month $(6.1$ [5.2 - 7.7] at 3 months, 6.3 [5.4 - 7.6] at 12 months; $p=0.001)$ for FPG respectively and 7.7 [6.9 - 9.7] at 3 months, 7.6 [6.6 10.7] at 12 months $p=0.001$ ) for PPG respectively).

Basically, HbA1c was 11.11 [9.8 - 12.3], in the 3rd month 6.5 [5.9 - 7.3], in the 12th month 6.31 [5.8 - 7.2]; treatment with SIIT significantly reduced $\mathrm{HbA1c}$ at $3^{\text {rd }}$ and $12^{\text {th }}$ month $(6.5$ [5.9 - 7.3] at 3 months, 6.31 [5.8 - 7.2] at 12 months $(p=0.001)$ for HbA1c. LDL-cholesterol level (mg / dl) was significantly reduced to $6.1[4.7-7.1]$ at 3 months and to 6.2 [5.1
Table-I: Baseline clinical and TANITA parameters.

\begin{tabular}{lc}
\hline Parameter & Baseline $n(65)$ \\
\hline Gender (F/M) & $24(36.90) / 41(63.07)$ \\
Age (year) & $48.03 \pm 10.78$ \\
Duration of DM (month) & $1.21 \pm 0.53$ \\
Height $(\mathrm{cm})$ & $165.35 \pm 10.09$ \\
Weight $(\mathrm{kg})$ & $83.32 \pm 17.42$ \\
BMI $\left(\mathrm{kg} / \mathrm{m}^{2}\right)$ & $30.48 \pm 5.81$ \\
TBMR $(\%)$ & $1630.43 \pm 245.72$ \\
T1FAT $(\%)$ & $30.83 \pm 10.87$ \\
FATMASS $(\mathrm{kg})$ & $30.83 \pm 10.87$ \\
TBW $(\mathrm{kg})$ & $40.42 \pm 7.31$ \\
FFM $(\mathrm{kg})$ & $55.21 \pm 9.98$ \\
\hline
\end{tabular}

DM: Diabetes Mellitus, BMI: Body Mass Index, TBMR: Total Basal Metabolisma Rate, T1 FAT: Total Body Fat Rate \%, FAT MASS: Total Body Fat Mass), TBW: Total Body Water, FFM: Fat-Free Body Mass.

- 7.5] at 12 months $(p=0.001)$ of SIIT therapy in 65 patients. The percentages of participants who had achieved their target $\mathrm{HbA1c}$ of $<7 \%$ were $67.3 \%$ after 3 months and $75 \%$ after 12 months.

Alanine aminotransferase (ALT) (U / L) basically 32.5 [24 - 53], 22.5 [16 - 29] at three months, 23.5 [18 - 37] at 12 months; IITD (month) 3 [1.5 - 3] at 3 months, 4 [2 - 6.5] at 12 months; LAITD (month)



Fig.1: Examination of FPG value by ROC analysis in predicting the cessation of insulin therapy and summary of analysis results. The ROC curve and reference diagonal line are shown together on the graph. 
Cengiz Karacaer et al.

Table-II: Comparisons of clinical characteristics between baseline, 2nd and 3rd visit.

\begin{tabular}{|c|c|c|c|c|}
\hline Parameters & $\begin{array}{c}\text { Baseline } \\
\text { [IQR:interquartile range] }\end{array}$ & $\begin{array}{c}\text { 2nd visit } \\
\text { (3rd month) } \\
\text { [IQR:interquartile range] }\end{array}$ & $\begin{array}{c}3 r d \text { visit } \\
(12 \text { month }) \\
\text { [IQR:interquartile range] }\end{array}$ & $p$ \\
\hline $\mathrm{N}$ & 65 & 57 & 65 & \\
\hline $\mathrm{FPG}(\mathrm{mmol} / \mathrm{L})$ & $15.3[10.7-18.8]$ & $6.1[5.2-7.7]$ & $6.3[5.5-7.6]$ & $<0.001^{\mathrm{a}, \mathrm{b}}$ \\
\hline PPG (mmol/L) & $19.7[14.8-23.3]$ & $7.7[6.9-9.7]$ & 7.6 [6.6- 10.7] & $<0.001^{\mathrm{a}, \mathrm{b}}$ \\
\hline HbA1c (\%) & $11.11[9.8-12.3]$ & $6.5[5.9-7.3]$ & $6.31[5.8-7.2]$ & $<0.001^{\mathrm{a}, \mathrm{b}}$ \\
\hline C-Peptide (pmol/ml) & $2.28[1.41-5.46]$ & $2.27[1.42-6.54]$ & $2.66[1.42-6.24]$ & 0.240 \\
\hline $\mathrm{LDL}(\mathrm{mg} / \mathrm{dl})$ & $7.1[5.7-9]$ & $6.1[4.7-7.1]$ & $6.2[5.1-7.5]$ & $0.001^{a, b}$ \\
\hline Creatinine $(\mathrm{mg} / \mathrm{dl})$ & $0.86[0.79-0.93]$ & $0.78[0.71-0.85]$ & $0.78[0.72-0.83]$ & $0.022^{b}$ \\
\hline $\operatorname{ALT}(\mathrm{U} / \mathrm{L})$ & $32.5[24-53]$ & $22.5[16-29]$ & $23.5[18$ - 37] & $<0.001^{a, b}$ \\
\hline SAI (IU, dose/day) & $24[24-30]$ & 26 [18 - 39] & $31[24-42]$ & 0.416 \\
\hline SAI (IU, dose/kg) & $0.33[0.26-0.39]$ & $0.35[0.23-0.43]$ & $0.4[0.29-0.47]$ & 0.841 \\
\hline LAI (IU, dose/day) & $10[10-14]$ & $12[10-16]$ & 14 [10 - 16] & 0.260 \\
\hline LAI (IU, dose/kg) & $0.13[0.11-0.18]$ & $0.15[0.11-0.2]$ & $0.16[0.13-0.21]$ & 0.382 \\
\hline TID (IU, dose/day) & $36[32-40]$ & 35 [24 - 49] & $35[18-50]$ & $0.003^{b}$ \\
\hline TID (IU, dose/kg) & $0.45[0.38-0.56]$ & $0.4[0.28-0.62]$ & $0.44[0.23-0.61]$ & $0.015^{b}$ \\
\hline IITD (month) & - & $3[1.5-3]$ & $4[2-6.5]$ & $<0.001^{\mathrm{a}, \mathrm{b}}$ \\
\hline LAITD (month) & - & $3[2-3]$ & $5[2-7.5]$ & $<0.001^{a, b}$ \\
\hline
\end{tabular}

FPG: Fast Plasma glucose, PPG: post-prandial glucose; HbA1c: glycated hemoglobin A1c; LDL: Low density lipoprotein; ALT: Alanine aminotransferase; SAI: Short acting insulin; LAI: Long acting insulin; TID: total insulin dose; IITD: Intensive insulin treatment duration, LAITD: Long acting insulin treatment duration. Multiple comparison test (Wilcoxon test with Bonferroni adjustment) results: There was statistically significant difference between; a: baseline and 3rd month, b: baseline and 3rd visit.

was found to be 3 [2 - 3] at 3 months, 5 [2 - 7.5] at 12 months $(p=0.001)$ (Table-II).

There was statistically significant positive correlation between delta total daily dose $(\triangle \mathrm{TDD})$ and fasting plasma glucose (FPG) (r: 0.418; p:0.003). There was statistically significant positive correlation between $\triangle \mathrm{TDD}$ and short acting insulin (SAI) (r: 0.532; p:0.001). Additionaly, it was statistically significant positive correlation between long-acting insulin (LAI) $(r=0.608 ; p=0.001)$. There were statistically significant negative correlation between total daily dose (TDD-1) and fasting plasma glucose (FPG)(r:-0.376; p:0.011) and also it were positive correlation between SAI and LAI(r:0.986; p:0.001) (r:0.928; p:0.001) (Table-III).

In the logistic regression analysis, it was found that the cessation of SIIT treatment after 3 months, FPG was an independent predictive factor $(p=0.015)$ at the first admission, but $\mathrm{HbA1c}$ and BMI were not significant predictors.

ROC analysis was performed to determine the optimal cut-off value of FPG for the determination of the requirement for insulin therapy beyond three months, and the area under the curve (AUC) was
Table-III: Correlation of TDD-1 or $\Delta$ TDD with baseline clinical and laboratory parameters.

\begin{tabular}{|c|c|c|c|c|}
\hline \multirow{2}{*}{ Parameters } & \multicolumn{2}{|c|}{$\triangle T D D(n=45)$} & \multicolumn{2}{|c|}{ TDD-1 $(n=45)$} \\
\hline & $r$ & $p$ & $r$ & $P$ \\
\hline Age & 0.169 & 0.250 & 0.311 & 0.375 \\
\hline Sex & -0.085 & 0.568 & 0.110 & 0.455 \\
\hline Weight (kg) & -0.114 & 0.441 & 0.218 & 0.137 \\
\hline BMI & -0.135 & 0.350 & 0.100 & 0.489 \\
\hline FPG & 0.418 & 0.003 & -0.376 & 0.011 \\
\hline $\operatorname{HgbA1c}(\%)$ & -0.036 & 0.807 & 0.090 & 0.545 \\
\hline LDL-c & -0.150 & 0.310 & -0.164 & 0.264 \\
\hline ALT & -0.208 & 0.166 & -0.009 & 0.953 \\
\hline Crea & -0.247 & 0.094 & -0.212 & 0.153 \\
\hline SAI & 0.532 & 0.001 & 0.986 & 0.001 \\
\hline LAI & 0.608 & 0.001 & 0.928 & 0.001 \\
\hline C-peptide & 0.010 & 0.960 & 0.247 & 0.959 \\
\hline
\end{tabular}

STDD: Delta total Daily dose, BMI: Body Mass Index, FPG: Fasting Plasma Glucose, HbA1c: glycated hemoglobin, LDL-c: Low density lipoprotein cholesterol; ALT: Alanine aminotransferase; Crea: Creatinine, SAI: Short acting insulin; TID: Total insulin dose; IITD: Intensive insulin treatment duration, LAITD: Long-acting insulin treatment duration. 


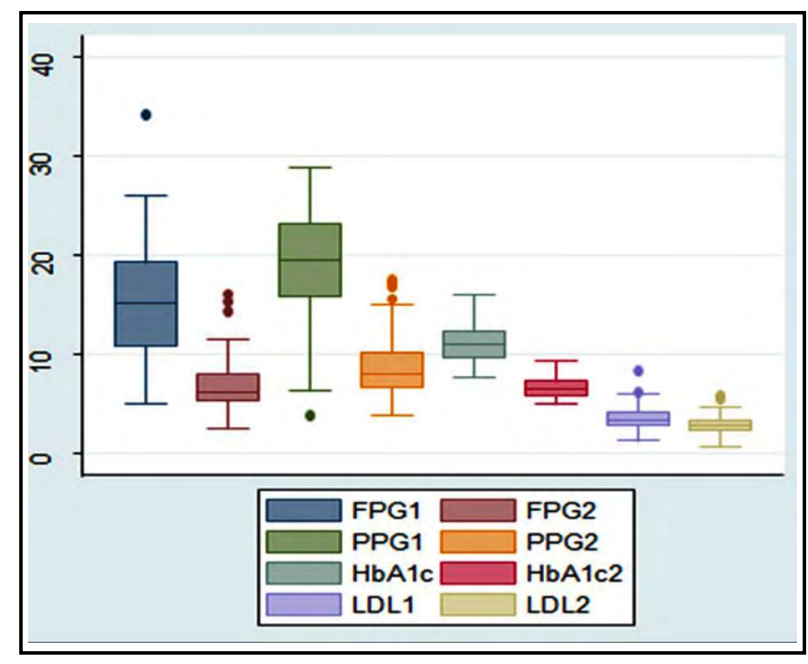

Fig.2: FPG1*-FPG2** , PPG1-PPG2, HbA1c1-HbA1c2, LDL1-LDL2 are proposed to be compared side-to-side(mmol/L).

FPG: Fasting Plasma Glucose, PPG: Post Prandial Glucose, LDL: Low Dansity Lipid *1: Initial values of pre-treatment, ${ }^{* *} 2$ : Third month values of treatment.

found to be significant (AUC: 0.694, 95\% CI: 0.557$0.831, p=0.021$ ). The use of FPG $\geq 13.8 \mathrm{mmol} / \mathrm{L}$ was associated with requirement for insulin therapy beyond three months and yielded a sensitivity of $62.5 \%$ and a specificity of $70.6 \%$ a LR analysis revealed that patients with FPG $>13.8 \mathrm{mmol} / \mathrm{L}$ were 7.6 times more likely to continue insulin therapy $(p=0.011)$ (Fig.1, Table-IV).

Comparisons of data between two time points were performed using the Wilcoxon test (Fig.2). These showed that there were significant differences in FPG, PPG, and LDL-cholesterol level between baseline and 3 months $(p=0.001)$ but that the C-peptide concentration did not differ $(p=0.558)$. There was also a significant difference in creatinine concentration between baseline and 12 months $(p=0.022)$.

The change in $\mathrm{HbA1c}$ during the first three months was close to significant following the application of the Bonferroni correction $(p=0.05)$. There were significant differences in serum creatinine, total insulin dose, and insulin dose per $\mathrm{kg}$ between baseline and the second and third visits (Table-III).

\section{DISCUSSION}

In the present study, we characterized the insulin requirements of $65 \mathrm{nT} 2 \mathrm{DM}$ patients who were treated using SIIT. The target HbA1c value after SIIT was achieved by $67.3 \%$ and $71.0 \%$ of participants after three months and 12 months,
Table-IV: Whether the insulin therapy as the categorical variable in the logistic regression analysis should be discontinued or not and the probability of continuing or discontinuing insulin when FBG $>250 \mathrm{mg} / \mathrm{dL}, \mathrm{BMI}$ and $\mathrm{HbA1c}$ are used as the independent predictor factors.

\begin{tabular}{lcccc}
\hline & $B$ & Odds Ratio & $95 \%$ C.I. & $P$ \\
\hline FPG & 0.011 & 1.011 & $1.002-1.020$ & 0.015 \\
BMI & 0.069 & 1.072 & $0.949-1.211$ & 0.266 \\
HBA1c & -0.192 & 0.825 & $0.552-1.235$ & 0.350 \\
\hline
\end{tabular}

FPG: Fast Plasma Glucose, BMI: Body Mass Index.

respectively. The initial insulin dose is $0.4-0.5$ units $/ \mathrm{kg}$, making 33.5-41.5 units for an average weight of $83-90 \mathrm{~kg}$. When euglycemia is reached, the daily insulin requirement is 36 [32-40] IU/ day at baseline, 35 [24-49] IU/day after three months, 35 [18-50] IU/day after 12 months, and a constant basal/bolus ratio of 2: It was 3. Although the average insulin doses do not appear to have decreased, it should be noted that the minimum insulin doses gradually decreased at the second and third visits. $(p=0.03)$ (Table-II).

To date, the proposed predictors of diabetic remission include the blood glucose concentration shortly after therapy, improvements in indices of beta-cell function, a reduction in insulin resistance, compliance with lifestyle modification, and a positive attitude towards the management of hyperglycemia. ${ }^{8,9}$

We have shown significant reductions in FPG, short acting insulin dose, and long acting insulin dose in correlation analyses performed using data from the $69 \%$ (45 of 65 ) of patients who had entered remission after 3 months of SIIT ( $p=0.011, p=0.001$, and $p=0.001$, respectively).In the study of Kramer CK et al. and Chen A et al., it were shown that shortterm intensive insulin therapy (SIIT) correlated with improvement in FPG in long-term outcomes in nT2DM patients. ${ }^{10,11}$

In the previous studies, FPG has been reported to have a predictive role in predicting the onset of diabetes, even if values are within the normal range. ${ }^{8,12-13}$ Ozery-Flato $\mathrm{M}$ et al. in their study, it was shown that they can accurately predict the development of T2DM with measurements such as BMI, FPG and HbA1c. ${ }^{14}$

In our study, although FPG lower than 13.8 $\mathrm{mmol} / \mathrm{L}$ is an independent predictive factor indicating that short term intensive insulin therapy can be cessation, BMI and HbA1c were not found to be an independent predictive factor. In 
previous studies, there are studies that reached this conclusion in a period of 6-12 months. ${ }^{15}$ There is a lack of consensus on the optimal duration of insulin therapy. Our study has provided information that the initial blood glucose can give an idea about the optimal duration of intensive insulin therapy in patients with newly diagnosed Type-2 diabetes who are metabolically glucotoxic.

Long-term insulin use has concerns regarding hypoglycemia, weight gain, decreased patient compliance and potential malignancy. ${ }^{16-18}$ This indicates that more effective predictive factors are needed to achieve glycemic control.

We found that FPG, PPG, HbA1c, LDLcholesterol, and ALT were significantly lower after 3 and 12 months than at baseline. However, no change in the $\mathrm{C}$-peptide concentration was detected $(p=0.240)$. Insulin action can be impaired by chronic hyperglycemia, but this defect can be ameliorated by the establishment of euglycemia. In the present study, an FPG of $9.2 \mathrm{mmol} / \mathrm{L}$, a PPG of $11.9 \mathrm{mmol} / \mathrm{L}$, and an HbA1c of $5.6 \%$ were found to be predictors of a recurrence of hyperglycemia (Table-II). We have shown that rapid correction of hyperglycemia can greatly improve $\beta$-cell function, especially with respect to first-phase insulin secretion. Therefore, in patients with nT2DM, in whom there has been a shorter duration of glucotoxicity and lipotoxicity, the early impairments in $\beta$-cell function can be reversed, which permits the long-term maintenance of glycemic control. In one study, $\mathrm{HbA1c}$ was significantly associated with various lipid parameters, and the importance of glycemic control in managing dyslipidemia and further reducing cardiovascular diseases risk in patients with T2DM was emphasized. ${ }^{19}$

SIIT has been used to obtain more rapid and better glycemic control in nT2DM patients, but predictors of long-term glycemic control have yet to be identified. Therefore, in nT2DM patients requiring insulin, we conducted a retrospective study that aimed to determine whether SIIT performed during the first 3 months after diagnosis would improve long-term glycemic control and ameliorate risk factors.

Limitations of the study: The major limitation of our study were lower sample size, retrospective design and the short-term follow-up period.

Strength of the study: It includes the high rate of improvement in FPG and the fact that this predictive factor was determined in the ideal period of 3 months is what makes it superior to other studies. The present study was designed to identify predictors of success in achieving glycemic control. However, the results require confirmation in larger studies before they can be applied in a routine clinical setting. Comparing the efficacy of intensive dietary therapy and aggressive therapy with insulin or OADs will be required in further studies.

\section{CONCLUSION}

The present study aimed to identify independent predictors of the need to prolong insulin therapy beyond three months. We found that FPG was an independent predictor in a model constructed using FPG, HbA1c, and BMI values very early in the course of nT2DM. $\beta$-cell function improved significantly during the first three months of SIIT, as did glycemic control. Our study shows that SIIT improves $\beta$-cell function and insulin sensitivity in patients with nT2DM.

Informed consent: Informed consent forms were obtained from all patients. Data were obtained for scientific purposes.

Conflicts of Interest: None.

Funding/Support Statement: None.

\section{REFERENCES}

1. Nathan DM. The diabetes control and complications trial/ epidemiology of diabetes interventions and complications study at 30 years: Overview. Diabetes Care. 2014;37(1):916. doi: 10.1089 /dia.2013.0081

2. Wang Y, Mendoza-Elias JE, Qi M, Harvat TA, Ahn SJ, Lee $\mathrm{D}$, et al. Implication of mitochondrial cytoprotection in human islet isolation and transplantation. Biochem Res Int. 2012;2012:16. doi: 10.1155/2012/395974

3. Yuan T, Yang T, Chen H, Fu D, Hu Y, Wang J, et al. New insights into oxidative stress and inflammation during diabetes mellitus-accelerated atherosclerosis. Redox Biol. 2019;20:247-260. doi: 10.1016/j.redox.2018.09.025

4. Leighton E, Sainsbury CA, Jones GC. A Practical Review of C-Peptide Testing in Diabetes. Diabetes Ther. 2017;8(3):475487. doi: 10.1007/s13300-017-0265-4

5. Nakashima K, Okamura N, Sanefuji H, Kaneto H. Practical application of short-term intensive insulin therapy based on the concept of "treat to target" to reduce hypoglycaemia in routine clinical site. Sci Rep. 2020;10(1):1-12. doi: 10.1038/s41598-020-58574-7

6. Hu Y, Li L, Xu Y, Yu T, Tong G, Huang H, et al. Short-term intensive therapy in newly diagnosed Type-2 diabetes partially restores both insulin sensitivity and $\beta$-cell function in subjects with long-term remission. Diabetes Care. 2011;34(8):1848-1853. doi: 10.2337/dc10-2105

7. Kramer CK, Zinman B, Retnakaran R. Short-term intensive insulin therapy in Type-2 diabetes mellitus: A systematic review and meta-analysis. Lancet Diabetes Endocrinol. 2013;1(1):28-34. doi: 10.1016/S2213-8587(13)70006-8 
8. Liu J, Liu J, Fang D, Liu L, Huang Z, Wan X, et al. Fasting plasma glucose after intensive insulin therapy predicted long-term glycemic control in newly diagnosed Type2 diabetic patients. Endocrine J. 2013;60(6):725-732. doi: 10.1507/endocrj.ej12-0315

9. Liu L, Yang S, Liu J, Li H, Liu J, Cao X, et al. Fasting plasma glucose indicates reversibility of the acute insulin response after short-term intensive insulin therapy in patients with various duration of Type-2 diabetes. J Diabet Res. 2018;2018:9423965. doi: 10.1155/2018/9423965

10. Kramer CK, Zinman B, Choi H, Retnakaran R. Predictors of sustained drug-free diabetes remission over 48 weeks following short-term intensive insulin therapy in early Type-2 diabetes. BMJ Open Diabet Res Care. 2016;4(1):e000270. doi: 10.1136/bmjdrc-2016-000270

11. Chen A, Huang Z, Wan X, Deng W, Wu J, Li L, et al. Attitudes toward diabetes affect maintenance of drugfree remission in patients with newly diagnosed Type-2 diabetes after short-term continuous subcutaneous insulin infusion treatment. Diabetes Care. 2012;35(3):474-481. doi: $10.2337 / \mathrm{dc} 11-1638$

12. Retnakaran $\mathrm{R}$, Zinman B. Short-term intensified insulin treatment in Type-2 diabetes: Longterm effects on $\beta$-cell function. Diabetes, Obesity Metabol. 2012;14(Suppl-3):161-166 doi: 10.1507/endocrj.EJ12-0315

13. Chon S, Oh S, Kim SW, Kim JW, Kim YS, Woo J Taek. The effect of early insulin therapy on pancreatic $\beta$-cell function and long-term glycemic control in newly diagnosed Type2 diabetic patients. KorJ Intern Med. 2010;25(3):273-281. doi: 10.3904/ kjim.2010.25.3.273

14. Ozery-Flato $M$, Parush N, El-Hay T, Visockiene $Z$, Ryliskyte L, Badariene J, et al. Predictive models for Type-2 diabetes onset in middle-aged subjects with the metabolic syndrome. Diabetol Metabolic Syndr. 2013;5(1):36 doi:10.1186/1758-5996-5-36
15. Hu Y, Li L, Xu Y, Yu T, Tong G, Huang H, et al. Short-term intensive therapy in newly diagnosed Type- 2 diabetes partially restores both insulin sensitivity and $\beta$-cell function in subjects with long-term remission. Diabetes Care. 2011;34(8):1848-1853. doi: 10.1186/1758-5996-5-36

16. But A, De Bruin ML, Bazelier MT, Hjellvik V, Andersen $\mathrm{M}$, Auvinen A, et al. Cancer risk among insulin users: comparing analogues with human insulin in the CARING five-country cohort study. Diabetologia. 2017;60(9):1691703. doi:10.1007/s00125-017-4312-5

17. Abudawood M. Diabetes and cancer: A comprehensive review. J Res Med Sci. 2019;24(1):94. doi:10.4103/jrms. JRMS $242 \_19$

18. Hosseini F, Mohammadbeigi A, Aghaali M, Borujerdi R, Parham M. Effect of pentoxifylline on diabetic distal polyneuropathy in Type-2 diabetic patients: A randomized trial. J Res Med Sci. 2019;24:89 doi:10.4103/ jrms.JRMS_115_18

19. Hussain A, Ali I, Ijaz M, Rahim A. Correlation between hemoglobin A1c and serum lipid profile in Afghani patients with Type-2 diabetes: hemoglobin A1c prognosticates dyslipidemia. Ther Adv Endocrinol Metab. 2017;8(4):5157. doi: $10.1177 / 2042018817692296$

\section{Author's Contribution:}

CK: Conceived the study, data collection, writing manuscript and responsible for integrity of study.

HC: Helped to draft the manuscript.

TD: Preparation of the manuscript.

$C V$ : Editing and final approval.

AT: Study design.

All authors read and approved the final manuscript.
Authors:

1. Dr. Cengiz Karacaer

Department of Internal Medicine

2. Taner Demirci

Assistant Professor,

Department of Endocrinology and Metabolism,

3. Dr. Hasret Cengiz

Department of Endocrinology and Metabolism,

4. Dr. Ceyhun Varim

Department of Internal Medicine

5. Ali Tamer

Professor,

Department of Internal Medicine,

1,4,5: Sakarya University Treatment and Research Hospital, Department of Internal Medicine, Sakarya, Turkey.

2,3: Sakarya University Treatment and Research Hospital, Department of Endocrinology and Metabolism, Sakarya, Turkey. 